\title{
Implementing a one-day testing model improves timeliness of workup for patients with lung cancer
}

\author{
M.A. Gulak BSCH, ${ }^{* \dagger}$ C. Bornais RN MN, ${ }^{\dagger}$ S. Shin $\mathrm{BA}_{,}^{+}$L. Murphy RSW MSW, ${ }^{\dagger}$ J. Smylie RN MHSM, ${ }^{+}$ \\ J.R. Pantarotto $\mathrm{MD}^{*+\neq}$ M. Fung-Kee-Fung MBBS $\mathrm{MBA}^{*}{ }^{*+\S}$ and D.E. Maziak MDCM MSc${ }^{*+\|}$
}

\begin{abstract}
Background Patients with lung cancer often experience stressful delays throughout the diagnostic phase of care. To address that situation, our multidisciplinary team created a "Navigation Day," during which patients partake in a single-day visit that comprises nurse-led teaching, social work, smoking cessation counselling, symptom control, and dedicated test slots for integrated positron-emission tomography and computed tomography (РET/CT), pulmonary function tests (PFTS), and magnetic resonance imaging (MRI) of the brain. We evaluated the effects of that program on wait times and patient satisfaction.
\end{abstract}

Methods Patients with a suspicion of lung cancer on chest cт imaging referred during 3 time periods were reviewed: 1 year before launch of the Navigation Day, 1 year post-launch, and 2 years post-launch. Patients were further stratified according to concordance of their test date with a Navigation Day date. Mean wait times for PET/CT, PFTs, and MRI brain were calculated for each group. Patient satisfaction was measured using a standardized provincial survey. The Student $t$-test and analysis of variance were used to assess for significance.

Results After implementation, mean wait times in the first year improved to 9.2 days from 15.5 days for PET/CT $(p<0.0001)$, to 9.6 days from 15.7 days for PFTs $(p<0.0001)$, and to 10.2 days from 16.0 days for MRI brain $(p<0.0001)$. Patients who used a dedicated test slot experienced the shortest wait times, at 5.8 days for PET/CT, 5.8 days for PFTs, and 6.3 days for MRI brain $(p<0.0001)$. Those improvements were sustained at 2 years post-launch. Patient satisfaction in the categories of assistance, emotional support, and clarity remained high post-launch.

Conclusions Navigation Day significantly improved the timeliness of diagnostic testing services in patients with suspected lung cancer.

Key Words Lung cancer, wait times, diagnostic delay, diagnostic testing, diagnostic assessment programs, clinical pathways, nurse navigators

Curr Oncol. 2019 October;26(5):e651-e657

www.current-oncology.com

\section{INTRODUCTION}

In Canada, lung cancer is the leading cause of cancer death in both men and women. It is responsible for the deaths of approximately 21,100 people annually, which is more than colorectal, breast, and prostate cancers combined ${ }^{1}$. Patients suspected of having lung cancer often go through a plethora of providers, examinations, delays, and specialists to obtain a diagnosis and treatment. This diagnostic phase of care can be an extremely anxiety-provoking and stressful experience, often with multiple appointments being needed and delays being encountered. Reducing those delays has been shown to relieve patient anxiety in patients suspected of having lung cancer ${ }^{2}$. Reducing wait times and the number of appointments to attend would therefore be highly beneficial for patients and their families.

The diagnostic phase of care in patients with lung cancer can include investigations such as integrated positron-emission tomography and computed tomography (PET/CT) imaging, pulmonary function tests (PFTs), magnetic 
resonance imaging (MRI) of the brain, and lung biopsy. Those diagnostic tests provide essential information for initiating stage-appropriate treatment. The timely delivery of a lung cancer diagnosis has therefore been an ongoing point of emphasis for centres worldwide. Nevertheless, a comprehensive review by Olsson et al. ${ }^{3}$ in 2009 revealed that time intervals to diagnosis and treatment are often longer than recommended by guidelines, a situation that has not changed in the time since ${ }^{4}$.

In 2014, The Ottawa Hospital (тон) defined delay to timely lung cancer care as a system-design problem and launched a transformation project to redesign lung cancer care with a patient-centred $\operatorname{model}^{5}$. As previously described, the model has 4 components that aim to improve delivery of care: people, process, technology, and a learning system $^{5}$. Using а тон-tailored business process management software application, those components of the endto-end process were standardized and streamlined, leading to the creation of a "Navigation Day" (Nav Day), during which patients and their family members are encouraged to partake in a day-long visit to receive coordinated testing and teaching. The Nav Day program aims to reduce wait times, reduce process variation, and enhance the overall experience for patients. Here, we report the effects of this unique lung cancer diagnostic service delivery model on wait times and patient satisfaction at тон.

\section{METHODS}

A multidisciplinary team piloted the Nav Day program for patients referred to the тон Diagnostic Assessment Program (DAP) for suspicion of lung cancer on chest CT imaging. Figure 1 depicts the thoracic intake process map for our centre.

All referrals are made through a central intake system and are reviewed every working day by a thoracic surgeon and thoracic radiologist at "Joint Review" to determine the appropriateness of the consultation and to begin the order set for the patient, based on the chest ст imaging. Joint Review is a process that was established at the same time as the Nav Day program as part of the transformation project. As described previously, it entails the use of numerous standardized processes, including physician order sheets, clinical patient notes, and physician duty rosters ${ }^{5}$. After Joint Review, appropriate patients are contacted by a nurse navigator for assessment and offered a Nav Day appointment (median time to first contact: 3 days; 90 th percentile: 5 days). At the time of contact, patients are also queried about their use of anticoagulant therapy to identify those who require a thrombosis consultation in preparation for their biopsy, a step that was implemented to avoid potential delays in timely diagnosis.

On their Nav Day, patients first undergo a group teaching session during which patients and their loved ones receive a program orientation from both a nurse navigator and a team social worker. Financial issues, commonly experienced emotions, and coping skills are discussed early on, facilitating open communication between the patient and staff. To mitigate any potential problems in the rapid completion of the diagnostic phase of care, each patient (and that patient's supporters) meets one-on-one with a nurse navigator who provides teaching about the upcoming testing and uses a self-reporting health history questionnaire and the Edmonton Symptom Assessment System to holistically assess symptoms, emotional status, and social issues. All necessary bloodwork is completed, smoking cessation teaching is initiated, contact numbers are reinforced for further questions, and a patient passport is provided and reviewed.

Before initiation of the Nav Day program, numerous handouts were available for patients with a diagnosis, but little information was available for patients still in the

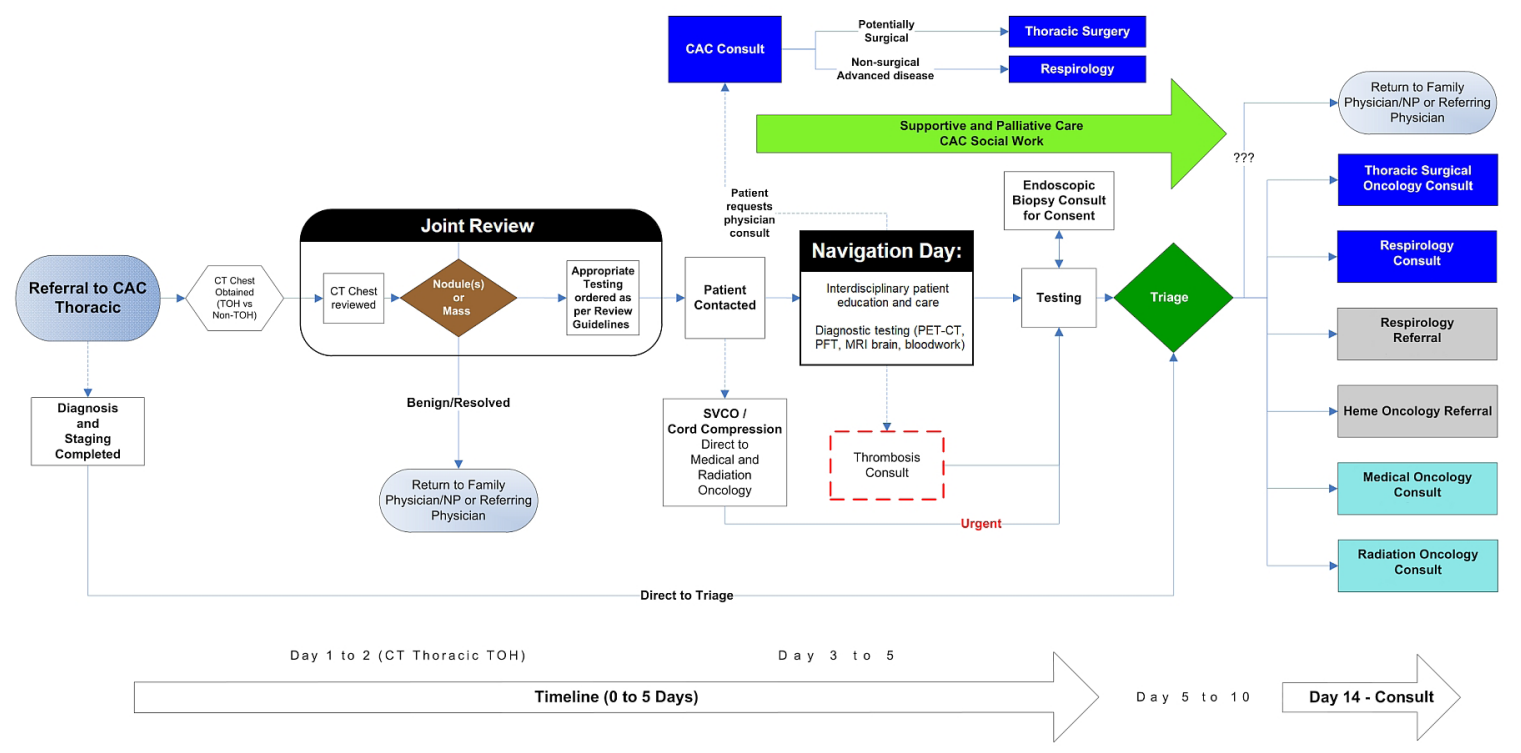

FIGURE 1 Overview of the thoracic intake process in the cancer Diagnostic Assessment Program at The Ottawa Hospital $(\mathrm{TOH})$. CT = computed tomography; NP = nurse practitioner; PET-CT = integrated positron-emission tomography and computed tomography; PFT = pulmonary function test; $\mathrm{MRI}=$ magnetic resonance imaging; $\mathrm{CAC}=$ Cancer Assessment Clinic (that is, the Diagnostic Assessment Program); SVCO = superior vena cava obstruction; ??? = no certainty of cancer. 
diagnostic phase of care. After a literature search and several focus group sessions that included former and current patients, the passport was created. The passport serves to familiarize patients with the testing being ordered and with the preparation for and locations of testing. It also contains calendar and medication sections, advice about how to find a family doctor for individuals without one, and suggestions for improving health status during this time. The purpose of the passport is to provide information that engages patients in becoming active in their own care.

A significant aspect of the Nav Day is its single-day diagnostic testing, which includes, as indicated, PET/CT imaging, PFTS, MRI brain, and bloodwork. Streamlined booking processes and dedicated testing slots for PET/CT, PFTS, and MRI brain were created in collaboration with respiratory therapy, nuclear medicine, and radiology colleagues for the Nav Day. With the exception of the biopsy, which occurs within 1 week of the Nav Day, all diagnostic testing required for staging and respiratory assessment can therefore be completed during the Nav Day.

The cohort for the present report includes all patients for whom PET/CT, PFTS, and MRIS were ordered at the time of Joint Review during 3 time periods relative to the implementation of the Nav Day: 1 year pre-launch, 1 year post-launch, and 2 years post-launch. Each period covered 8 months for PET/CT and PFTs (November 2013 to June 2014, November 2014 to June 2015, and November 2015 to June 2016) and 6 months for MRI brain (April 2014 to September 2014, April 2015 to September 2015, and April 2016 to September 2016). Nav Day-dedicated test slots for MRI were established at a later date than those for PET/CT and PFTS (April 2015 vs. November 2014), and so the chosen periods ensure that wait times immediately after implementation were captured, while maintaining the distinction of the "pre-launch" and "post-launch" periods. Mean, median, and 90th percentile wait times were calculated for each test type, measured as the number of days from the date of the request to the date of the procedure. Wait times included holidays and weekends even if no testing was done on those days. Because some patients did not use their dedicated Nav Day slot, patients were further stratified by whether their procedures were performed on Nav Day or outside of Nav Day, thus specifically assessing the effect on wait times of having dedicated spots (5 PET/CT, 4 PFTs, and 1 MRI brain per Nav Day, with 3 Nav Days per week). The data were captured by a tailored clinical process management тон Web portal system and a DAP-Thoracic database (Access: Microsoft Corporation, Redmond, WA, U.S.A.). All data were merged into the DAP-Thoracic database and queried based on the foregoing criteria. Descriptive data were generated using a spreadsheet (Excel: Microsoft Corporation). Student $t$-tests and 2-way analyses of variance were used to assess for significance in the reduction of mean wait times. All statistics were 2 -sided, and a $p$ value less than 0.05 was considered statistically significant. The statistical analyses were performed in the GraphPad Prism software application (version 6.01: GraphPad Software, La Jolla, CA, U.S.A.).

Patient satisfaction with Nav Day was measured by examining data from the Patient Experience Survey distributed by Cancer Care Ontario. The survey is a standardized, anonymous questionnaire that captures the patient experience during the diagnostic process at all DAPs in Ontario. Survey questions were amalgamated in groups of 3-4 into three broad categories: assistance of health care workers, emotional support, and clarity in understanding the diagnostic tests (Table I). Responses were converted from "agree" descriptors into a 1-5 scoring system to allow for comparison. Patient satisfaction in those categories as expressed by all respondents was compared between the pre-launch and 1 year post-launch periods at тон, and with satisfaction as expressed by respondents from all DAPs in the province. Specialized surveys distributed to patients at тон were also used to quantify patient satisfaction in the 1 year post-launch period. Those surveys were given to patients after they had completed their Nav Day, received the appropriate testing, and been triaged and seen by the appropriate service to review the results, thereby capturing patient satisfaction encompassing the entire diagnostic phase of care.

\section{RESULTS}

\section{Effect of the Nav Day Program on Wait Times}

In the 3 defined periods (1 year pre-launch, 1 year postlaunch, and 2 years post-launch), 363, 455, and $480 \mathrm{PET} /$ Ст imaging tests; 316, 360, and 377 PFTs; and 219, 228, 192 MRI brain were performed. The mean wait time for each test type improved significantly from 1 year pre-launch to 1 year post-launch $(p<0.0001$ for each test type, by $t$-test; Figure 2). Specifically, the mean wait time declined by 6.3 days for PET/CT (to 9.2 days from 15.5 days, $40.7 \%$ reduction), by 6.1 days for PFTs (to 9.6 days from 15.7 days, $39.0 \%$ reduction), and by 5.8 days for MRI brain (to 10.2 days from 16.0 days, 36.3\% reduction; Figure 2). Further improvements in mean wait time from 1 year post-launch to 2 years postlaunch were also seen for all tests, and the difference was statistically significant for MRI brain $(p<0.0001)$.

From 1 year pre-launch to 1 year post-launch, median wait times were also reduced: by 7.4 days for PET/CT (to 6.6 days from 14.0 days, $52.9 \%$ reduction), 7.5 days for PFTs (to 6.5 days from 14.0 days, $53.6 \%$ reduction), and 8.4 days for MRI brain (to 7.6 days from 16.0 days, $52.5 \%$ reduction). The 90th percentile wait times were reduced by 6.5 days for PET/ Ст (to 17.5 days from 24.0 days, $27.0 \%$ reduction), 10.8 days for PFTs (to 19.7 days from 30.5 days, $35.5 \%$ reduction), and 7.4 days for MRI brain (to 18.6 days from 26.0 days, $28.4 \%$ reduction). All of those improvements were sustained in the 2 years post-launch period.

Improvements in wait time dispersion also occurred for each test type from 1 year pre-launch to 2 years postlaunch, as shown by reductions in the standard deviations by 1.1 days for PET/CT (to 8.1 days from 9.6 days, $11.2 \%$ reduction), 1.1 days for PFTs (to 9.9 days from 11.1 days, $10.1 \%$ reduction), and 1.9 days for MRI brain (to 7.8 days from 9.7 days, $19.1 \%$ reduction).

\section{Effect of Dedicated Test Slots on Wait Times}

To determine the effect of the dedicated test slots on wait times, patients who underwent each test in the 1 year postlaunch period were stratified according to whether they underwent their test on their Nav Day or on a day different from their Nav Day, or whether they did not attend a Nav 
TABLE I Scores on the Cancer Care Ontario patient satisfaction survey in the Navigation Day pre-launch and 1 year post-launch periods for the Ottawa Diagnostic Assessment Program (DAP) and for all Ontario DAPs

\begin{tabular}{|c|c|c|c|c|c|}
\hline \multirow[t]{3}{*}{ Category } & \multirow[t]{3}{*}{ Survey question } & \multicolumn{4}{|c|}{ Score out of $5^{a}$} \\
\hline & & \multicolumn{2}{|c|}{ Ottawa DAP } & \multicolumn{2}{|c|}{ Ontario DAPs } \\
\hline & & Pre-launch & Post-launch & Pre-launch & Post-launch \\
\hline 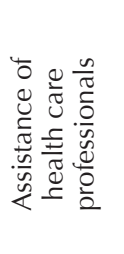 & $\begin{array}{l}\text { During the diagnostic process ... } \\
\text { The health care team gave me instructions on how to get ready for } \\
\text { my next appointment. } \\
\text { The health care team answered my questions or connected me to } \\
\text { someone who could. } \\
\text { The health care team knew where I was in the diagnostic process. } \\
\text { The Navigator dealt with questions or concerns I had. }\end{array}$ & 4.76 & 4.79 & 4.64 & 4.76 \\
\hline 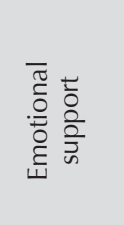 & $\begin{array}{l}\text { During the diagnostic process ... } \\
\text { I was comfortable talking about my worries/concerns with the health } \\
\text { care team. } \\
\text { I felt supported when I was told my diagnosis/that I needed } \\
\text { treatment/that I did not need any more testing at that time. } \\
\text { The Navigator helped me cope with the diagnostic process. }\end{array}$ & 4.72 & 4.69 & 4.57 & 4.67 \\
\hline 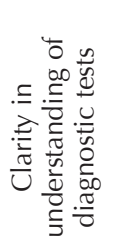 & $\begin{array}{l}\text { During the diagnostic process ... } \\
\text { The health care team told me why I needed tests in a way that I } \\
\text { could understand. } \\
\text { The doctor told me about my test results in a way that I could } \\
\text { understand. } \\
\text { The Navigator helped me understand the tests I was going to have. } \\
\text { The Navigator explained to me their role in my care. }\end{array}$ & 4.77 & 4.75 & 4.65 & 4.77 \\
\hline
\end{tabular}

a Responses were converted from "agree" descriptors to a 1-5 scoring system (1, Strongly disagree; 2, Somewhat disagree; 3, Neither; 4, Somewhat agree; 5, Strongly agree) and were averaged in each category for the purposes of this comparison.

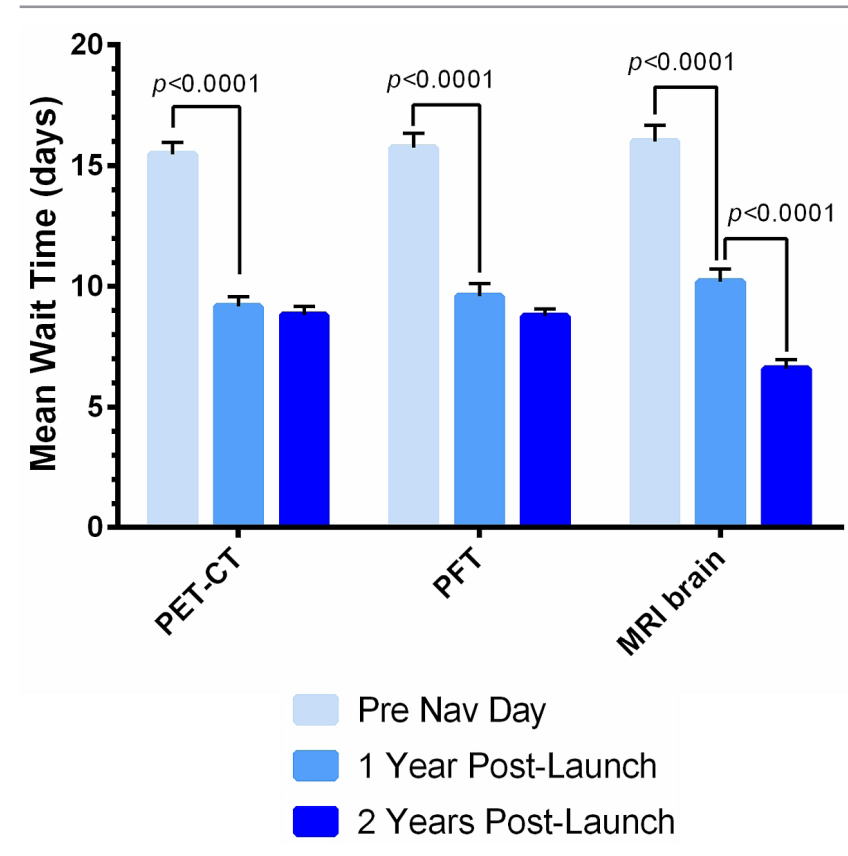

FIGURE 2 Summary of mean wait times for integrated positron-emission tomography and computed tomography (PET-CT), pulmonary function test (PFT), and magnetic resonance imaging (MRI) of the brain. Nav Day = Navigation Day.
Day (Figure 3). Patients who underwent tests on their Nav Day (that is, using a dedicated slot) experienced the shortest wait times for all tests. They also experienced significantly shorter wait times than patients whose Nav Day and test dates were discordant: 5.8 days compared with 15.5 days for PET/Ст ( $p<0.0001, t$-test), 5.8 days compared with 15.2 days for PFTs $(p<0.0001)$, and 6.3 days compared with 10.7 days for MRI brain $(p<0.0001)$. That effect of test date on wait time was also found to be significant by 1-way analysis of variance $(p<0.0001)$.

Some reasons that patients had a scheduled Nav Day, but a different test day were being scheduled but not attending, being unable to complete the procedure on Nav Day, unavailability of a procedure slot on Nav Day, or not meeting the criteria for the procedure at the time. Patients who did not have a Nav Day scheduled at all said that they were feeling too rushed or that they wanted to see a specialist before undergoing any testing.

\section{Patient Satisfaction}

Examination of patient satisfaction was conducted for тон and for all DAPS in the province of Ontario in 3 broad categories: assistance of health care professionals, emotional support, and clarity in understanding the diagnostic tests (Table I). In the 1 year post-launch period, тон demonstrated scores comparable to its pre-launch scores $(4.79,4.69$, and 4.75 vs. $4.76,4.72$, and 4.77 respectively), indicating 


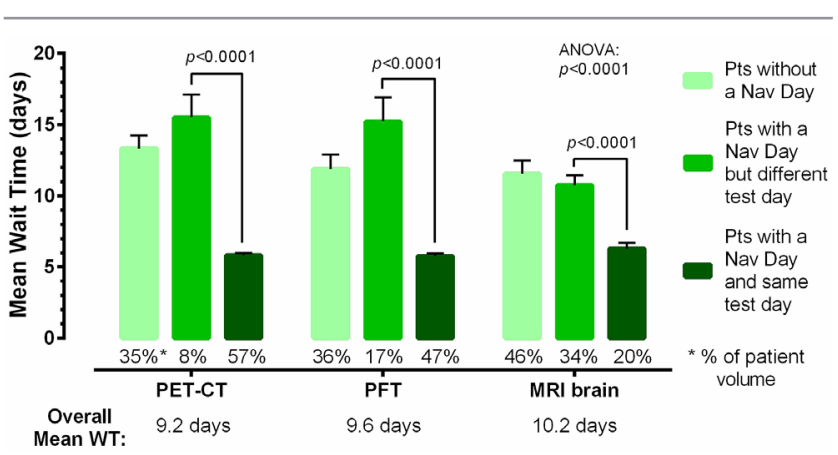

FIGURE 3 Mean wait times (WT) for integrated positron-emission tomography and computed tomography (PET-CT), pulmonary function test (PFT), and magnetic resonance imaging (MRI) of the brain in the 1 year post-launch period, stratified by test date. Pts = patients; Nav Day = Navigation Day.

preservation of patient satisfaction after program implementation. In addition, тоH was on par with provincial standards, with scores comparable to those for all DAPs in the province of Ontario (4.76, 4.67, and 4.77 respectively).

Patient satisfaction was also measured using a specialized 5-question survey distributed after the specialist consultation (and answered by 38 patients), with $97 \%$ agreeing that their tests were well coordinated, $95 \%$ agreeing that their tests were done quickly enough, $92 \%$ agreeing that they knew those to contact if they had questions, $92 \%$ agreeing that the passport was helpful, and $96 \%$ agreeing that they had the opportunity to ask questions.

\section{DISCUSSION}

The Nav Day program was implemented as part of a much larger transformation project involving significant regional process redesign that aimed to improve timely access to lung cancer diagnosis for a significant catchment area in eastern Ontario ${ }^{5}$. In Ontario, health care administration is organized into 14 geographic regions called Local Health Integration Networks (LHINs) which fund and coordinate the health care services delivered by hospitals and other public health care centres within their area. The Ottawa Hospital falls within the administration of the Champlain LHIN, which services approximately 1.3 million residents across 18,000 square kilometers. In the Champlain LHIN, тон is the largest-and only_-thoracic cancer intake centre, having provided new-patient consultations for approximately 900 patients with lung cancer in the 2014-2015 fiscal year (1 year post-launch). Previously published work highlighted the improvements arising from the regional process redesign, with тон ranking 1 st among the 14 regional cancer centres in providing patients with timely lung cancer diagnosis ${ }^{5}$. More specifically, the target set by Cancer Care Ontario was that $65 \%$ of patients should receive a diagnosis within 28 days of referral, and in the 2015-2016 fiscal year (2 years post-launch), the percentage of patients in the Champlain LHIN diagnosed within the target 28 days was $80 \%$ compared with $57 \%$ for the other LHINs in the province of Ontario ${ }^{5}$. The performance in the Champlain region after the transformation was statistically improved compared with the province as a whole and with the region before the transformation. Here, we show the specific improvements in wait times from referral to PET/CT (to 8.8 days from 15.5 days), PFTs (to 8.8 days from 15.7 days), and MRI brain (to 6.6 days from 16.0 days) over a 2 -year period that played a significant role in allowing тон to exceed the provincial targets for timely lung cancer diagnosis.

It is still unclear whether delays in diagnosis and treatment affect patient outcomes; studies show mixed and sometimes paradoxical results ${ }^{3,4}$. Nevertheless, many centres in North America and Europe have successfully used interventions such as care navigators and rapid diagnostic assessment programs to improve wait times for patients with lung cancer. However, the wait time most commonly reported and targeted is the time from lung cancer diagnosis to initial treatment ${ }^{4}$, and studies that address delays specifically in the diagnostic phase of care are fewer ${ }^{2,6,7}$. Given that the diagnostic phase of care can be an extremely distressing period for patients suspected of having lung cancer ${ }^{8}$ and that arriving at a diagnosis more quickly can reduce patient anxiety ${ }^{9}$, shortening any diagnostic delays is favourable. In fact, one observational study by Brocken et al. ${ }^{2}$ in 2015 demonstrated that patients suspected to have lung cancer experience less distress in a rapid diagnostic program than in a standard stepwise program. Our Nav Day program is an effort to reduce diagnostic delays through the integration of centralized intake, nurse navigator-led care, regular multidisciplinary meetings, and standardized diagnostic algorithms, which share similarities with other programs $s^{6,7,10}$. However, we are the first to report the novel implementation of dedicated testing slots for PET/CT, PFTS, and MRI brain and to demonstrate significant improvement and sustainability in wait times for those tests. Given the importance of PET/ $\mathrm{CT}^{11}$ and MRI brain ${ }^{12}$ results for clinical decision-making, timeliness and availability of those tests for patients who might have lung cancer cannot be understated.

Beyond benefits for wait times, the strengths of the Nav Day program include its multidisciplinary approach, emphasis on patient-centred care, nurse-led care coordination, and avoidance of the need for multiple specialist appointments. The introductory group teaching session with the nurse navigator and team social worker addresses financial issues, coping strategies, and commonly experienced emotions, facilitating open communication between staff, patients, and patient supporters early on. The subsequent one-on-one nurse assessment allows for smoking cessation to be addressed as early as possible. Additionally, the patient passport created for the program not only eases a patient's transition into care with easy-to-understand information, but engages them to be an active participant in their care, with sections that make suggestions for health improvement and how to find a family physician. For patients who already have a primary care provider, ongoing communication is established to ensure transparency of the patient's care. Furthermore, the model of nurse-led care coordination means that the nurse navigators have a central role in developing relationships with patients. They assume the role of the "go-to" person for patients, providing ongoing support for questions and issues that might arise throughout the patient's journey. The relationship begins early, because patient contact with the nurse navigator 
is established quickly, the median time from referral to scheduling of the Nav Day appointment being 3 days (90th percentile: 5 days). Finally, the program reduces the burden of specialist appointments on patients in two ways: First, before Nav Day, the intervening daily Joint Review process allows for plans to be made before the patient's first contact with a specialist. Second, after Nav Day, patients are immediately triaged and given stage-appropriate referrals to medical oncology, radiation oncology, a combined appointment, or thoracic surgery (Figure 1). Those features, in addition to the "one-stop shop" approach of diagnostic testing, ensures that no wasted appointments are ever scheduled as the patient moves through the diagnostic phase of care.

Our study is not without its limitations. Arguably, similar wait time reductions might have been achieved with the implementation of dedicated test slots without a Nav Day. And yet, patients in the 1-year post-launch period who attended a Nav Day, but who did not use a dedicated test slot, experienced shorter wait times than did patients in the pre-launch period (data not shown), suggesting that the Nav Day program itself, likely partly because of the Joint Review process, contributed to wait-time reduction to some degree. Nonetheless, we emphasize that the Nav Day program encompasses numerous other beneficial aspects as already highlighted. With those benefits in mind, we acknowledge that patient satisfaction did not improve as much as might have been expected; however, the data extracted from provincial surveys might underestimate post-launch patient satisfaction given that the survey questions do not necessarily pertain specifically to Nav Day, and respondents would not have experienced both the pre- and post-launch diagnostic processes.

Another limitation is that the retrospective design of the study meant that some data were incomplete. For example, voluntary compared with involuntary reasons for not using the dedicated test slots or not having a Nav Day at all could be distinguished only to a limited extent. A disproportionate number of patients were seen to undergo MRI brain, but not a Nav Day (46\%), and $35 \%$ of patients had a Nav Day, but underwent MRI brain on a different day. That observation is explained by the fact that MRI brain tests are ordered only for patients with suspected or confirmed advanced disease, and the point at which advanced status is determined in the diagnostic flow would dictate whether a patient is excluded from a Nav Day (in most cases, patients with advanced status at the time of referral would be excluded, with the exception of those having Pancoast disease) or attends a Nav Day, but undergoes MRI brain on a different day (patients whose Nav Day PET/CT suggests advanced disease). Regardless, some patients still voluntarily refused their Nav Day appointment, often citing feeling too rushed or wanting to see a physician first.

Another limitation is that the time from referral to tissue diagnosis was not reported in the present study. However, our standard is that all lung biopsies are conducted within 1 week of the Nav Day, and previously published work already highlights our centre's leading performance in the province for timely diagnosis ${ }^{5}$. Because the present study was conducted at a single university-affiliated tertiary care centre, we acknowledge that the results might be affected by referral bias and that our model might not be applicable to smaller tertiary care centres with limited access to resources such as PET/CT, MRI brain, and lung biopsy. Lastly, whether our demonstrated improvement in timeliness of diagnostic testing services translates to improved clinical outcomes has yet to be seen, although the value of shorter diagnostic delays in reducing emotional distress in patients with suspected lung cancer has previously been shown ${ }^{2}$.

\section{CONCLUSIONS}

Implementation of a Nav Day program and dedicated test slots significantly improved the timeliness of diagnostic testing services (PET/CT, PFTs, and MRI brain) while maintaining a high level of satisfaction for patients suspected to have lung cancer. This model of single-day expedited diagnostic testing and multidisciplinary care could serve as a model for other centres that serve patients with lung cancer and other malignancies.

\section{ACKNOWLEDGMENTS}

We acknowledge the entire team of the Ages Cancer Assessment Clinic at тон who make the Nav Day model a reality on a daily basis. Some of these data were presented at the International Association for the Study of Lung Cancer's 19th World Conference on Lung Cancer; 23-26 September 2018; Toronto, ON.

\section{CONFLICT OF INTEREST DISCLOSURES}

We have read and understood Current Oncology's policy on disclosing conflicts of interest, and we declare that we have none.

\section{AUTHOR AFFILIATIONS}

*Faculty of Medicine, University of Ottawa, Ottawa, ON; ${ }^{\dagger}$ The Ottawa Hospital Research Institute, and ${ }^{\ddagger}$ Division of Radiation Oncology, ${ }^{\circledR}$ Division of Gynecologic Oncology, and $\|_{\text {Division of }}$ Thoracic Surgery, The Ottawa Hospital, Ottawa, ON.

\section{REFERENCES}

1. Canadian Cancer Society's Advisory Committee on Cancer Statistics. Canadian Cancer Statistics 2018. Toronto, ON: Canadian Cancer Society; 2018.

2. Brocken P, van der Heijden EH, Oud KT, et al. Distress in suspected lung cancer patients following rapid and standard diagnostic programs: a prospective observational study. Psychooncology 2015;24:433-41.

3. Olsson JK, Schultz EM, Gould MK. Timeliness of care in patients with lung cancer: a systematic review. Thorax 2009;64:749-56.

4. Jacobsen MM, Silverstein SC, Quinn M, et al. Timeliness of access to lung cancer diagnosis and treatment: a scoping literature review. Lung Cancer 2017;112:156-64.

5. Fung-Kee-Fung M, Maziak DE, Pantarotto JR, et al. Regional process redesign of lung cancer care: a learning health system pilot project. Curr Oncol 2018;25:59-66.

6. Alsamarai S, Yao X, Cain HC, et al. The effect of a lung cancer care coordination program on timeliness of care. Clin Lung Cancer 2013;14:527-34.

7. Common JL, Mariathas HH, Parsons K, et al. Reducing wait time for lung cancer diagnosis and treatment: impact of a multidisciplinary, centralized referral program. Can Assoc Radiol J 2018;69:322-7.

8. Brocken P, Prins JB, Dekhuijzen PNR, van der Heijden HFM. The faster the better? A systematic review on distress in the diagnostic phase of suspected cancer, and the influence of rapid diagnostic pathways. Psychooncology 2012;21:1-10. 
9. Moseholm E, Rydahl-Hansen S, Overgaard D, et al. Healthrelated quality of life, anxiety and depression in the diagnostic phase of suspected cancer, and the influence of diagnosis. Health Qual Life Outcomes 2016;14:80.

10. Lo DS, Zeldin RA, Skrastins R, et al. Time to treat: a system redesign focusing on decreasing the time from suspicion of lung cancer to diagnosis. J Thorac Oncol 2007;2:1001-6.
11. Subedi N, Scarsbrook A, Darby M, Korde K, Mc Shane P, Muers MF. The clinical impact of integrated FDG PET-CT on management decisions in patients with lung cancer. Lung Cancer 2009;64:301-7.

12. Schellinger PD, Meinck HM, Thron A. Diagnostic accuracy of MRI compared to СCT in patients with brain metastases. $J$ Neurooncol 1999;44:275-81. 